Anuario Latinoamericano Ciencias Políticas

y Relaciones Internacionales

vol. 7, 2019

pp. 353-355

\section{Thomas Piketty, El capital en el siglo XXI, Cambridge, Massachusetts, London 2014, pp. 685, ISBN 978-0-674-43000-6}

\author{
Thomas Piketty, Capital in the Twenty-First Century, \\ Cambridge, Massachusetts, London 2014, pp. 685, ISBN \\ 978-0-674-43000-6
}

Una de las obras más relevantes y discutidas en los círculos académicos y políticos a nivel internacional, dedicadas al tema de la riqueza y la desigualdad, ha sido sin duda Capital de Thomas Piketty. El libro publicado en 2014 se basa en datos de los que se tiene registro durante tres siglos para las economías de Francia, Reino Unido, Alemania y los Estados Unidos. Se divide en cuatro apartados y dieciséis capítulos. El primer apartado se titula Ingreso y Capital; el segundo: El dinamismo de la relación Capital/Ingreso; el tercero: La estructura de la desigualdad, y el cuarto: El capital en el siglo veintiuno.

En el primero destaca que, junto a la existencia de sistemas políticos que privilegian la propiedad privada, la creciente concentración de la riqueza en las naciones desarrolladas se explica por dos tipos de desaceleración: demográfica y de productividad. Para entender este fenómeno es necesario analizar la evolución de la relación capital/ingreso, es decir, la relación entre el acervo total de capital y el flujo anual del ingreso. El ingreso es una variable de flujo y representa la cantidad de bienes producidos y distribuidos en un año. El acervo de capital corresponde a la riqueza total en un determinado periodo. Éste puede ser resultado de una apropiación o de un proceso de acumulación a través del tiempo. La forma más natural y útil para medir el acervo de capital en un país es dividiendo su acervo de capital entre el flujo de su ingreso anual, con lo cual obtenemos la relación capital/ingreso, representada con la letra griega beta $\beta$. Por ejemplo, si el acervo de capital de una nación equivale a seis años de su ingreso nacional, diremos que: $\beta=6$. En las naciones industrializadas la relación capital/ingreso oscila entre 5 y 6 , siendo el acervo de capital mayoritariamente privado. En seguida define la primera ley fundamental del capitalismo a través de la siguiente fórmula: $\alpha=r^{*} \beta$, donde $\alpha$ (alfa) equivale a la participación del ingreso derivado del capital en el ingreso nacional y donde $r$ es la tasa de retorno del capital. Por ejemplo, si beta equivale a seis veces el ingreso y el rendimiento es del 5\%, entonces la participación del capital en el ingreso (alfa) es igual a 30\%. Es decir: $r=5 \% ; \beta=600 \%$, $\alpha=30 \%$ 
$\left(r * \beta=0.05^{\star} 600\right)$. La fórmula es una identidad contable pura y es aplicable a cualquier economía en cualquier periodo de la historia. Es fundamental porque expresa los tres conceptos más relevantes del sistema capitalista: a) la relación capital/ingreso; b) la participación del capital en el ingreso y c) la tasa de retorno del capital. Si bien la tasa de retorno del capital puede generar rendimientos por arriba del 10\% -en bienes raíces se han observado niveles de entre 3-4\%; pero también ha habido periodos en que ha resultado negativa-, la tasa de rendimiento de largo plazo en el acervo de capital ha oscilado entre el 7\% y $8 \%$. En 2010, la fórmula fundamental aplicada arrojaba una alfa estimada de $30 \%$; una beta de $600 \%$ y una $r$ de $5 \%$. Ello se traduce en un ingreso per cápita de 30 mil euros. De ese monto, 21 mil euros corresponden a la mano de obra y 9 mil al capital. Piketty calcula que a cada individuo le corresponden 180 mil euros de capital, que le generan un rendimiento de 9 mil euros al año, es decir el 5\%.

En el siguiente apartado el autor presenta la segunda ley fundamental del capitalismo que consiste en $\beta=s / g$, donde $\beta$ es la relación capital/ingreso, $s$ la tasa de ahorro y $g$ la tasa de crecimiento del ingreso per cápita. Una economía que ahorra el $12 \%$ de su ingreso y presenta una tasa de crecimiento del $2 \%$ arrojará en el largo plazo una relación capital/ingreso de $600 \%$ o seis veces su ingreso nacional. Es decir, una economía que ahorra mucho y crece lentamente acumulará a largo plazo un enorme acervo de capital, fenómeno que tendrá repercusiones en la estructura y distribución de la riqueza. Piketty muestra que la $\beta$ mundial de 1870 a 2100 acusa un crecimiento constante hacia finales del siglo XIX que alcanza los 500\% hasta 1910, para comenzar un descenso que toca fondo hacia 1950 con menos de $300 \%$, reflejo de las secuelas de las dos guerras mundiales; a partir de ahí sigue un tendencia ascendente, ayudada por tasas de crecimiento menores que caen de un $3 \%$ promedio a $1,5 \%$ en la segunda mitad del siglo XXI; asumiendo una estabilización de la tasa de ahorro de $10 \%, \beta$ podría alcanzar los $700 \%$ para 2100 . Con una $\beta$ de $700-800 \%$, la tasa de retorno de capital $r$ podría oscilar entre $4-5 \%$ y la participación del capital en el ingreso total se situaría entre 30-40\%.

En la tercera parte Piketty habla sobre la estructura de la desigualdad. Explica que existen tres tipos de desigualdad: a) la derivada del ingreso laboral, b) la vinculada con propiedad del capital y su rendimiento, y c) la que resulta de la interacción entre ambas. Basándose en la popular novela de Balzac, Papa Goriot, Piketty extrae un pasaje en donde una de las figuras de nombre Vautrin, afirma que es ilusorio pensar que la riqueza material pueda alcanzarse mediante el esfuerzo, el talento o el estudio; a ello le denomina: "la enseñanza de Vautrin". Por ello apunta que la riqueza heredada o la que surge por medio del matrimonio ha jugado un papel fundamental a lo largo de la historia, principalmente en la Europa Occidental del siglo XIX y hasta el término de la Segunda Guerra Mundial; después, el estudio y el trabajo también se convirtieron en las rutas hacia el éxito. La desigualdad en la propiedad del capital es mucho mayor en comparación con la del trabajo. Este fenómeno se observa permanentemente a lo largo de la historia. El 10\% de los trabajadores más ricos reciben entre 25-30\% del ingreso 
laboral; mientras que el $10 \%$ de los propietarios del capital más ricos reciben $50 \%$ del ingreso del capital. A quienes viven de los rendimientos del capital, el autor los denomina rentiers. Piketty advierte que, hasta lo que va del siglo XXI, presenciamos una transición de una sociedad de rentiers a una de supermanagers; se trata de personas que perciben muy altos salarios, fenómeno que se ha hecho notorio principalmente en los Estados Unidos. Piketty explica que el factor demográfico aumentará la concentración de la riqueza por el lado del capital, ya que en sociedades en donde la población se contrae, a menor número de individuos corresponderá mayor riqueza.

En el siglo XXI, las fuerzas de la desigualdad provienen tanto del capital por el efecto de la herencia y cambios demográficos, por un lado, como por el trabajo, a través del fenómeno de supermanagers, por el otro; ambos enriqueciéndose en comparación con los que menos tienen. El grupo de los supermanagers no siempre accede a la riqueza por medio de la meritocracia, sino también por la suerte y el robo. Se percibe entonces una desigualdad creciente y sin aparente freno. Por ello, en el cuarto apartado analiza el problema de la tributación al capital. Afirma que todo intento de fijar impuestos a las grandes fortunas para atenuar la desigualdad ha fracasado por la existencia de los paraísos fiscales y el secreto bancario: “The most plausible reason why tax havens defend bank secrecy is that it allows their clients to evade their fiscal obligations, thereby, allowing tax havens to share in the gains" (521). Piketty propone un impuesto global al capital; una política fiscal global que impida la fuga de capitales ante el menor intento de cobrarles impuestos; ello a la par de la implementación de una base de datos bancaria internacional accesible a todos los sistemas financieros para evitar la evasión fiscal.

Piketty concluye que, si se deja la economía de mercado operar libremente, continuará alimentando dos fuerzas: convergente y divergente. La primera consiste en la difusión del conocimiento y las bondades de la tecnología en beneficio de la sociedad; la segunda terminará minando las bases de la democracia liberal y con ello, aniquilando los valores de la justicia social. Este proceso se impulsa a través de la desigualdad $r>g$, que significa que el rendimiento del capital por efecto de la herencia supera al del crecimiento del ingreso per cápita. Ello se traduce en una apropiación de la riqueza creciente a manos de los que más tienen en comparación con los que menos tienen; $\mathrm{o}$, en otras palabras, el capital termina por reproducirse más rápido de lo que la economía crece: el pasado devora al futuro. Este fenómeno no es nuevo; se dio a finales del siglo XIX y se desarticuló durante el siglo XX a causa de dos guerras mundiales. De ahí que pensar que el impuesto global al capital pueda ser una solución para evitar una próxima catástrofe.

Gerardo Reyes Guzmán

Facultad de Negocios, Universidad DeLaSalle Bajío Campus Salamanca, Guanajuato, México

$\triangle$ gerardo1960@yahoo.com https://orcid.org/0000-0001-9637-942X
Anuario Latinoamericano Ciencias Políticas y Relaciones Internacionales vol. 7, 2019

pp. 353-355

DOI: 10.17951/al.2019.7.353-355 